\title{
Analysis of Filament Arrangements and Generation of Statistically Equivalent
}

\section{Composite Micro-Structures}

F. Gommer ${ }^{*}$, A. Endruweit, A.C. Long

Polymer Composites Group - Division of Materials, Mechanics \& Structures, University of Nottingham, University Park, Nottingham NG7 2RD, UK

* Corresponding author (F.Gommer@ nottingham.ac.uk)

\begin{abstract}
An efficient method to describe and quantify the filament arrangement in fibre bundles based on the analysis of micrographs was developed. Quantitative measurement of relative filament positions indicated that the initially random arrangement of filaments shows increasingly strong characteristics of square and hexagonal configurations with increasing level of transverse compaction. An existing micro-structure generator was extended to incorporate the measured data allowing statistically equivalent filament arrangements to be generated at any fibre volume fraction. These can be used to determine micro-structural properties of actual fibre reinforced composites.
\end{abstract}

Keywords: A. Carbon fibres, C. Statistics, C. Modelling, D. Optical microscopy

\section{Introduction}

Mechanical and processing properties of fibre reinforced composites are strongly influenced by the heterogeneity of the reinforcement. During impregnation of the reinforcement with a liquid resin system, the variability in the filament arrangement leads to local differences in flow velocities and an increased probability of air entrapment which in turn may reduce the service life of the finished part [1]. In addition, published studies showed that the mechanical properties are overestimated if the random filament arrangement at the micro-scale is disregarded [2]. Most material 
models incorporating micro-scale variability of the filament arrangement are based on artificially generated random configurations [3]. These models do, however, not necessarily exhibit the same statistical filament distribution as present within actual fibre bundles. Owing to the difficulty of gathering data on the intrinsic variability of fibre reinforcements [4], algorithms which can generate statistically equivalent microstructures are rarely implemented. This study describes an automated method for determination of parameters to describe the micro-structure of a fibre bundle and statistical analysis of the measured data. In addition, an algorithm is proposed to generate equivalent representative micro-structure models which is adapted from the work of Vaughan and McCarthy [5]. These models can be used to predict reinforcement processing properties, e.g. resin flow during impregnation in composites processing, and micro-scale properties of composite parts.

\section{Materials and Data Acquisition}

To characterise the degree of heterogeneity in fibre bundles at different transverse compression levels, single layer composite panels were moulded and cured at different cavity heights. Panels with dimensions $125 \mathrm{~mm} \times 60 \mathrm{~mm}$ were made from a low-crimp fabric and a low viscosity epoxy resin system. The fabric consisted of uni-directional carbon fibre bundles, stabilized by thin glass weft threads coated with a thermoplastic polymer (Fig. 1).

The specimens were moulded by circumferential injection of the liquid resin into a stiff metallic tool containing the fabric. By varying the mould cavity height, $h$, the level of fabric compression was controlled and three sets of panels with global fibre volume fraction, $V_{f}$ of $0.45,0.60$ and 0.74 were produced [6]. The influence of bundle compaction on the micro-structure was then analysed based on a total of 26 fibre bundle 
cross-sections, extracted at random positions.

The micro-structure of moulded and cured specimens was determined for entire fibre bundles at high resolution by means of optical microscopy. Other imaging techniques, such as micro-computed tomography [7], were identified to have inferior resolution. Images were acquired with a brightfield reflected light microscope equipped with an automated stage and a 12 bit monochrome camera. The pixel spacing, i.e. the distance between pixel centres, of the resulting images was determined to be $0.093 \mu \mathrm{m}$. This resolution was considered to be sufficient and the method is inexpensive and simple compared to, for example, a Scanning Electron Microscope (SEM).

Large area scanning was enabled by a computer-controlled stage, which allows automated $x$-y positioning as well as automated $z$-positioning of the sample. Stitching of overlapping single images enables large areas to be studied [8,9]. A stitching code was implemented based on Fast-Fourier correlations [10] programmed in Matlab ${ }^{\circledR}$. This allowed the micrographic analysis of entire fibre bundles in cross-sections of the manufactured composite panels. The stitched images were analysed automatically at much higher resolution compared to examples reported in the literature [11].

\section{Image Analysis}

To gain morphological data from the acquired images, a simple colour thresholding technique is often employed $[8,12]$. However, inevitable differences in lighting, local light reflections and shading [13] make it unfeasible to use a single global threshold for a complete image which can contain up to 250 individual filaments. This effect is amplified locally by the presence or absence of filament cross-sections within the image. This results in different greyscale values for the same object. Fig. 2A shows a section of a captured 8 bit micrograph. The greyscale values are re-coloured in Fig. 2B 
to emphasise local colour differences of the filament cross-sections.

In addition, the theoretical resolution of an optical light microscope is limited by the wavelength of the light used and can be estimated as about $0.2 \mu \mathrm{m}$ for visible light [13]. It is impossible to distinguish between object edges which are closer to each other than the resolution limit. Employing a greyscale threshold to Fig. 2 and watershed separation of the remaining objects [14] will result in filament edges which are not determined correctly (Fig. 3). For example, areas of adjacent filament cross-sections appear to belong to another filament, leading to erroneous morphological data.

Owing to the problems encountered when a simple thresholding technique is employed, an automated image analysis process was developed in this study. This analysis is based on the edge detection of local colour gradients and overcomes the issues described above. This allows the micro-structure of a fibre bundle containing a large number of filaments to be determined accurately in an automated manner.

\subsection{Image Processing}

Since the simple thresholding technique cannot be applied for accurate detection of filament cross-sections, a different approach is utilised here, based on prior knowledge of the expected filament shape. For the case of carbon filaments a circular crosssectional shape is presumed, which may appear elliptical if the filament axis is not normal to the cross-sectional plane [15]. A window of defined size is centred on every single filament cross-section estimated by simple global thresholding and a watershed separation [14], and localised image analysis is executed. It is possible to detect filament edges directly in these local images using established algorithms, e.g. Canny's edge detector [16]. Employing this method (Fig. 4A) and fitting an ellipse to the detected edges, it was found, however, that the detected filament radii are 
underestimated compared to the dimensions of a set of manually fitted ellipses on high magnification SEM images (Fig. 4B).

Instead of using the original image, local colour gradients were calculated (Fig. 4C) in these locally confined areas. This allows absolute colour values of the image to be neglected. The problem of different levels of contrast or brightness, which depends on the position of the filament in the overall image, can therefore be overcome. After smoothing the image, edges of the colour gradients were determined using Canny's edge detector. The image of the filament boundary gradients shows two distinct edges (Fig. 4D). Utilising the results in Fig. 4A, the inner edges are eliminated, which leaves just the outer filament edge of interest and noise arising from edges of neighbouring filament cross-sections. This noise can be removed by selecting points of the edges closest to the image centre only. The resulting ellipse fit (Fig. 4E) can be optimised by iteratively removing outliers with a specified offset to this ellipse (Fig. 4F).

Compared to a set of 29 manually fitted ellipses on SEM images (Fig. 4B), an average underestimation of $0.8 \%$ of the minor ellipse axis was identified employing the method described above. The measurement of 300,000 single filament cross-sections showed a normal distribution of the filament diameter with a mean of $6.97 \mu \mathrm{m}$ and a standard deviation of $0.39 \mu \mathrm{m}$, which is in good agreement with the nominal manufactures' values. The precision of these results is superior to measurement data reported in the literature $[5,15,17]$. The exact determination of the filament cross-sectional shape also allows precise estimation of the entire fibre bundle micro-structure, e.g. the relative filament (centre) positions.

The presumed elliptical fit of the filament boundary is applicable to carbon fibres but may be questionable for the cross-sections of other (irregular shaped) filaments, in 
particular natural fibres [18]. However, the proposed process allows the use of any other shape approximation, making it a powerful tool to systematically analyse large areas within a composite at high magnification.

\section{Results}

For the produced samples with the respective global $V_{f}$ of $0.45,0.60$ and 0.74 (Section 2), a total of 9, 10 and 7 fibre bundles were analysed. The analysed bundles contained a total of 102590, 112557 and 80581 individual filament cross-sections, respectively. The results presented in this section describe the micro-structure of the entire fibre bundles. The description of local variabilities found within fibre bundles [19] is not analysed in this work.

\subsection{Bundle Shape}

Based on the method to accurately determine the dimensions and the exact location of the filaments described in Section 3.1, it is possible to deduce the edge of the entire fibre bundle. The average width of individual fibre bundles, $w$, increases significantly in the initial stages of through-thickness bundle compression $\left(V_{f}=0.45\right.$ to 0.60$)$ and remains almost constant if the bundle is compressed further (Fig. 5). Due to the increase in $w$, adjacent bundles overlap for $V_{f}=0.60 \mathrm{~mm}$ and merge for $V_{f}=0.74 \mathrm{~mm}$. Only in areas where the fixation thread is present, the yarns remain physically separated. The significant change of the fibre bundle shape when compacted from $V_{f}=0.45$ to 0.60 suggests that the $V_{f}$ within the fibre bundle should be less affected. Only if the bundle is further compressed (from $V_{f}=0.60$ to 0.74 ), the $V_{f}$ within the bundle should significantly increase due to the limited change in bundle dimensions.

\subsection{Micro-Structure}

\subsubsection{Nearest Neighbour Distances}


Different statistical descriptors can be employed to describe the random arrangement of filaments within a fibre bundle [20,21]. The distance, $l$, measured between the centres of neighbouring filaments can give an idea of the spatial arrangement of the microstructure [22]. It was found that measured distances of a filament to its $n$-th nearest neighbour exhibit a right-tailed distribution which could be approximated by a lognormal probability density function (Fig. 6). The distributions also exhibit a lower truncation limit since two filaments cannot interpenetrate one another. The minimum possible distance between two filaments is therefore equivalent to the sum of their radii. To allow comparison between different samples, the distances of a filament to its $n$-th nearest neighbour were normalised by the respective average filament diameter, $d_{\text {avg }}$, of the sample.

For the analysed $V_{f}$, the filament distances between closest neighbours $(n=1)$ show similar distributions (Fig. 6). Only a slight reduction of the mean value is observed when $V_{f}$ is increased. The narrow distributions and peak values close to 1 indicate that the most probable distance between closest filaments is the average filament diameter. This implies that filaments are touching. Because double counts of distances between filaments were not avoided, smaller filaments are likely to be the closest neighbour to more than one filament. This can explain the distribution peak values which are slightly below 1, suggesting that the distance between the majority of closest filaments is smaller than the average filament diameter.

With an increase in $n$ (Fig. 6; $n=2,3$, and 4), the distributions get wider, indicating a larger spread in distances between filaments. In addition, the sample mean becomes larger which suggest a lower probably of touching filaments. Whilst the average distances between filaments increase with increasing $n$, it was found that the positive 
skew gets more pronounced only until approximately $n=6$ for all $V_{f}$. The distribution shape remains constant for neighbouring filaments of $n>6$.

With increasing $V_{f}$, the $n$-th neighbour distance distributions narrow and the location $\left(l / d_{\text {avg }}\right)$ of the peak value decreases. This behaviour is more distinct for the second, third and fourth nearest neighbour distance distributions (Fig. 6; $n=2,3$, and 4). The change of the peak value implies that the average distance between filaments decreases with an increasing level of compaction. The narrower distributions reflect the increasing packing density of the filaments and are directly related to an increasingly uniform filament arrangement. Furthermore, the decrease in inter-filament distance is more pronounced when the fibre bundle is compacted from 0.60 to 0.74 global $V_{f}$ within the mould. This indicates that, due to a change in yarn shape (Section 4.1), inter-filament distances decrease less significantly when compacted from 0.45 to 0.60 global $V_{f}$.

\subsubsection{Nearest Neighbour Angles}

The distance to the $n$-th nearest neighbour indicates that with an increase in compaction of the fibre bundle, the inter-filament distance becomes more uniform. The angle, under which the $n$-th nearest neighbouring filament is located, should also reflect this increased degree of order within the fibre bundle but is often disregarded. For analysis of the samples, the $0^{\circ}$ direction was chosen to be aligned with the width of the fibre bundle and hence, $\pm 90^{\circ}$ represents the direction of the sample thickness.

It can be demonstrated that the distribution of angles, under which the $n$-th nearest neighbours are located, changes significantly with increasing fabric compaction. With minimal fabric compaction, the distribution of angles is almost random (Fig. 7A). A minor preference of the closest neighbour in direction of the bundle width $\left(0^{\circ} / 180^{\circ}\right)$ can be observed. This could result from the fabric production process when the fibre 
tow is flattened by pulling through various loops before being processed into the textile reinforcement and/or the fabric storage in the form of a tightly wound roll. The angle distributions become, however, less uniform with increasing compaction (Fig. 7B and C), i.e. there is an increasing degree of order in the filament arrangement.

With an increase in $V_{f}$, the probability for the closest filament $(n=1)$ to be found in the compaction direction $\left( \pm 90^{\circ}\right)$ is significantly increased as a result of the bundle compaction (Fig. 7B). The results shown in Fig. 6 suggest that these filaments are most likely in contact. In addition, due to the decrease in cavity height, filaments are pushed in the direction normal to the compaction. An increased probability of the third and fourth nearest neighbour to be located in the direction of the bundle width results. This effect can be observed as bundle widening in Fig. 5. A further increase in bundle compaction does lead to a limited increase of bundle width only due to the reduced available space (Fig. $5 V_{f}=0.74$ ). Therefore the filaments need to rearrange more significantly at the micro-scale. The increased probability for a filament's $n$-th neighbour to be located at angles of $0^{\circ}, 60^{\circ}, 120^{\circ}, 180^{\circ}, 240^{\circ}$ and $300^{\circ}$ (Fig. 7C) indicates that the filament arrangement approaches hexagonal packing.

Misalignment of the angle distributions with respect to the direction of the bundle width and height can be observed. This effect is more distinct in the measured angle distribution of the sample with maximum compaction (Fig. 7C). The most probable location of the first neighbouring filament is at an angle of $120^{\circ}$ or $300^{\circ}$ respectively. It can be speculated that the onset of this preference of the angles results from the mould closing process. A slight misalignment of the upper and lower mould tools would lead to slightly misaligned compaction planes. This effect could be further pronounced when physically compacting the fibre reinforcement in the mould. An increased compaction 
force could have been introduced from one side of the mould, which would explain the preference for a certain angle for the (first) nearest neighbour position in Fig. 7.

\section{Micro-Structure Generation}

For modelling of micro-structural properties, i.e. reconstruction of filament arrangements at the micro-scale, several models have been proposed in the literature. The most simple method of generating random filament arrangements places nonoverlapping disks representing the filaments with constant $[23,24]$ or varying [25] radii randomly within a specified domain. A limitation to this method is the jamming limit at around $V_{f}^{\text {loc }}=0.55[26,27]$, which is at the lower end of the typical local $V_{f}$ within fibre bundles used in composite materials. Other studies induce randomness by disturbing regular [28] or random arrangements in defined ways [29, 30]. Varying numbers of stirring iterations can be employed to achieve different degrees of order [31]. Employing these techniques, artificial filament arrangements with $V_{f}$ up to the theoretical maximum packing can be generated. However, each of these modelling approaches lacks a direct correlation to quantified micro-structures. This limitation is overcome by the modelling approach proposed by Vaughan and McCarthy [5], which uses measured filament distributions.

\subsection{Reconstruction of Filament Arrangements}

Statistically equivalent two-dimensional micro-structures were generated following an adapted version of the procedure described by Vaughan and McCarthy [5] by means of measured distributions of distances between neighbouring filaments. In their model, a pre-determined number of filaments are placed at distances picked randomly from measured distributions, and in random directions relative to a randomly placed starting 
filament. After the last filament is placed, the starting point is moved to the first newly placed filament, and the process is repeated. These steps are repeated until the specified domain is filled with filaments, and no additional filament can be placed.

For periodic hexagonal and square filament arrangements, the maximum $V_{f}$ is achieved if the nearest neighbour distance is equal to the constant filament diameter $\left(V_{f}^{\text {loc }} \approx 0.91\right.$ and $\approx 0.79$, respectively). It can be demonstrated, however, that the procedure described by Vaughan and McCarthy has a natural jamming limit of $V_{f}^{\text {loc }} \approx 0.70$ if only neighbour distances are used and varying numbers of filaments are placed in the reconstruction of the filament arrangement (Fig. 8). This limit can be explained by the fact that, whilst the neighbouring filament distance is picked from a measured distribution, the neighbouring filament angle is randomly assigned. This introduces an unintended assumption about the arrangement which does not necessarily reflect the actual filament configuration (Fig. 9A).

Therefore, the proposed algorithm [5] was developed further to utilise the measurement data presented in Section 4.2. The adjustment of the nearest neighbour distributions to avoid double counts of distances between filaments which are each other's $n$-th nearest neighbour was omitted. This adjustment would effectively lead to a widening of the measured distributions. Allowing double counts does, however, reflect the reality where two filaments within one yarn cross-section can be each other's $n$-th nearest neighbour. To accommodate the change in procedure, the first four nearest neighbour distributions were used for the filament placement procedure (Fig. 6). In addition, the filament radii were picked from a normal distribution as measured for the actual fibre bundles in Section 3.1. 
As indicated in Section 4.2, the filament arrangement becomes more uniform with increasing levels of compaction. Therefore, it is necessary to take the actual angle distributions into account at which the $n$-th nearest filament is located rather than using a randomly assigned angle. Incorporating this consideration into the procedure proposed by Vaughan and McCarthy, a statistically equivalent filament arrangement can be generated. By utilising the filament distance and angle distributions for periodic hexagonal packing with maximum $V_{f}^{\text {loc }} \approx 0.91$, the actual filament arrangement can be reconstructed (Fig. 9B). This demonstrates that the jamming limit of the fibre placement algorithm as shown in Fig. 9A can be overcome by using the modified procedure proposed here.

\subsection{Validation of Generated Arrangements}

The generated micro-structural arrangements of square domains with a length to fibre radius ratio of 43 were validated with the same statistical descriptors as employed earlier for a large number of realisations. The distances of a filament to its $n$-th nearest neighbour exhibit a similar trend as the measured log-normal mean for the three $V_{f}$ analysed (Fig. 10). It can be seen that the spread of the generated neighbour distances are well within the confidence interval of one (log-normal) standard deviation displayed as grey shaded areas. These distributions are, however, more narrow than the experimentally determined distributions. It is thought that this results from the smaller domain size generated compared to the entire fibre bundles. This therefore disregards local anomalies found within real fibre bundles such as bundle separations. In addition, edge effects such as a reduced number of filaments at the bundle tips or locally confined areas of low filament densities within the bundles are also not likely to be recreated.

\section{Concluding Remarks}


This study proposes a new method to quantify and describe the filament arrangement within entire fibre bundles of reinforcement fabrics for composites at the micro-scale. Morphological data of individual filaments were gained from micrographs of fibre bundle cross-sections employing edge detection on determined local colour gradients. This method overcomes issues commonly found when employing conventional image analysis techniques such as simple colour thresholding. The detected filament outlines are approximated with an ellipse which, for the case of carbon fibres, was concluded to be a good fit. The analysis method was implemented in Matlab ${ }^{\circledR}$ and allowed the systematic study of entire fibre bundles at high image resolution. The measurement of a large number of single filament cross-sections showed a normal distribution of the filament diameter which is in good agreement with nominal values. It was concluded that the developed automated measurement technique delivers more accurate results compared to measurement data presented previously in the literature.

The distance of a filament to its $n$-th nearest neighbour was selected to describe the spatial arrangement of the filaments. For the analysed single-layer composite samples it was shown that these distances to nearest neighbours decrease with increasing fibre bundle compaction. In the literature, the increasing degree of order of the filament arrangement, reflected in the angle distribution under which the $n$-th nearest neighbour is located, is disregarded. The obtained results indicate that the initially random filament arrangement within a fibre bundle shows tendencies to become square under initial and hexagonal under high level of compression. It should be stressed, however, that these are tendencies only and the filaments are still not arranged in an ideal periodic pattern. The analysis of the angle distribution in this work was only made possible because of the developed automated measurement technique that allows a systematic analysis of a 
large number of images. The trends of changing micro-structures observed in this work need to be confirmed for other materials.

To generate statistically equivalent micro-structures, a previously proposed fibre placement algorithm was further developed to utilise the presented data. In particular, the incorporation of the angle distribution of nearest neighbours guaranteed a statistically equivalent model and also eliminated the observed jamming limit of $V_{f}^{\text {loc } \approx}$ 0.70. It is now possible to generate any $V_{f}$ depending on the provided input data. In combination with the proposed image analysis method to measure the statistical filament arrangement, the developed micro-structure generator can, for example, be used for the prediction of micro-mechanical or processing properties of composites.

\section{Acknowledgements}

This work was supported by the Engineering and Physical Sciences Research Council [grant number: EP/IO33513/1], through the EPSRC Centre for Innovative Manufacturing in Composites (CIMComp) and by an EPSRC Doctoral Prize award hosted by the University of Nottingham.

\section{References}

[1] Chambers AR, Earl JS, Squires CA, Suhot MA. The effect of voids on the flexural fatigue performance of unidirectional carbon fibre composites developed for wind turbine applications. Int J Fatigue. 2006;28:1389-1398.

[2] Trias D, Costa J, Mayugo JA, Hurtado JE. Random models versus periodic models for fibre reinforced composites. Comput Mater Sci. 2006;38(2):316-324.

[3] Yazdchi K, Srivastava S, Luding S. Micro-macro relations for flow through random arrays of cylinders. Compos Part A. 2012;43(11):2007-2020. 
[4] Paluch B. Analysis of geometric imperfections affecting the fibers in unidirectional composites. J Compos Mater. 1996;30(4):454-485.

[5] Vaughan TJ, McCarthy CT. A combined experimental-numerical approach for generating statistically equivalent fibre distributions for high strength laminated composite materials. Compos Sci Technol. 2009;70(2):291-297.

[6] Gommer F, Endruweit A, Long AC. Generation, Modelling and validation of statistically equivalent micro-structures Generation. 19th International Conference on Composite Materials (ICCM19), Montreal, Canada; 2013.

[7] Requena G, Fiedler G, Seiser B, Degischer P, Di Michiel M, Buslaps T. 3DQuantification of the distribution of continuous fibres in unidirectionally reinforced composites. Compos Part A. 2009;40(2):152-163.

[8] Eberhardt C, Clarke A. Fibre-orientation measurements in short-glass-fibre composites. Part I: automated, high-angular-resolution measurement by confocal microscopy. Compos Sci Technol. 2001;61(10):1389-1400.

[9] Davidson NC, Clarke AR, Archenhold G. Large-area, high-resolution image analysis of composite materials. J Microsc. 1997;185(2):233-242.

[10] Luong B. FFT based convolution. www.mathworks.co.uk. Last accessed 15.4.2013 [11] Zangenberg J, Larsen JB, Østergaard RC, Brøndsted P. Methodology for characterisation of glass fibre composite architecture. Plast Rubber Compos. 2012;41(45):187-193.

[12] Gajdosík J, Zeman J, Sejnoha M. Qualitative analysis of fiber composite microstructure: Influence of boundary conditions. Probabilist Eng Mech. 2006;21(4):317-329. 
[13] Wu Q, Merchant FA, Castleman KR. Microscope Image processing. London: Elsevier Inc.; 2008.

[14] Lotufo RA, Audigier R, Saúde AV, Machado RC. Morphological Image

Processing. In: Wu Q, Merchant FA, Castleman KR, editors. Microscope Image Processing: Elsevier Inc.; 2008. p. 113 - 157.

[15] Blanc R, Germain C, costa JPD, Baylou P, Cataldi M. Fiber orientation measurements in composite materials. Compos Part A. 2006;37(2):197-206.

[16] Russ JC. The Image Processing Handbook. Fifth Edition. Broken Sound Parkway NW: CRC Press; 2007.

[17] Summerscales J, Russel PM. Observations on the fibre distribution and fibre strain in a woven fabric reinforcement. Adv Compos Letters. 2004;13(3):135-140.

[18] Kratmann KK, Sutcliffe MPF, Lilleheden LT, Pyrz R, Thomsen OT. A novel image analysis procedure for measuring fibre misalignment in unidirectional fibre composites. Compos Sci Technol. 2009;69(2):228-238.

[19] Silberschmidt VV. Effect of micro-randomness on macroscopic properties and fracture of laminates. J Mater Sci. 2006;41(20):6768-6776.

[20] de Azeredo Lopes SM. Statistical Analysis of Particle Distributions in Composite Materials PhD thesis. University of Sheffield, 2000.

[21] Stoyan D, Stoyan H. Fractals, random shapes and point fields - Methods of geometrical statistics: John Wiley \& Sons; 1995.

[22] Gommer F, Endruweit A, Long AC. Non-uniformity of the filament distribution in fibre bundles and its effect on defect formation in liquid composite moulding. 18th International Conference on Composite Materials (ICCM18), Jeju Island, South Korea; 2011. 
[23] Buryachenko VA, Pagano NJ, Kim RY, Spowart JE. Quantitative description and numerical simulation of random microstructures of composites and their effective elastic moduli. Int J Solids Struct. 2003;40(1):47-72.

[24] Chen X, Papathanasiou TD. Micro-scale modeling of axial flow through unidirectional disordered fiber arrays. Compos Sci Technol. 2007;67(7-8):1286-1293. [25] Gusev AA, Hine PJ, Ward IM. Fiber packing and elastic properties of a transversely random unidirectional glass/epoxy composite. Compos Sci Technol. 2000;60(4):535-541.

[26] Nakanishi Y, Matsumoto K, Kurashiki T, Zako M. Multiscale analysis of material damping properties for textile composites. 13th European Conference on Composite Materials (ECCM13), Stockholm; 2008.

[27] Zhou E, Mollenhauer D, Iarve E. A realistic 3-D textile geometric model. 17th International Conference on Composite Materials (ICCM17), Edinburgh; 2009.

[28] Wongsto A, Li S. Micromechanical FE analysis of UD fibre-reinforced composites with fibres distributed at random over the transverse cross-section. Compos Part A. 2005;36(9):1246-1266.

[29] Cai Z, Berdichevsky AL. Numerical simulation on the permeability variations of a fiber assembly. Polym Compos. 1993;14(6):529-539.

[30] Sangani AS, Yao C. Transport processes in random arrays of cylinders. II. Viscous flow. Phys Fluids. 1988;31(9):2435-2444.

[31] Bechtold G, Ye L. Influence of fibre distribution on the transverse flow permeability in fibre bundles. Compos Sci Technol. 2003;63(14):2069-2079. 


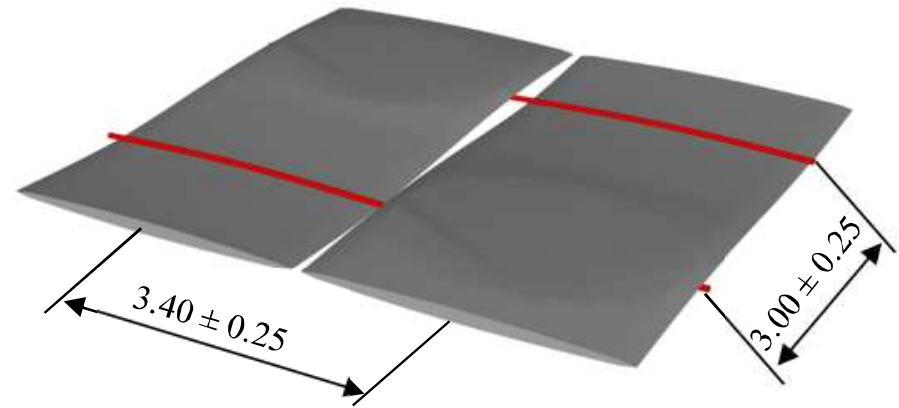

Fig. 1. Idealised unit cell of the quasi UD fabric. Fabric data provided by Sigmatex Ltd.: Areal density, $A_{f}$ : $300 \mathrm{~g} / \mathrm{m}^{2}$, Warp yarns: T700SC 12K 50C, Weft yarns: Fusible combi-yarn (225Dtex). Dimensions shown are in mm. Image created with TexGen available from http://texgen.sourceforge.net.

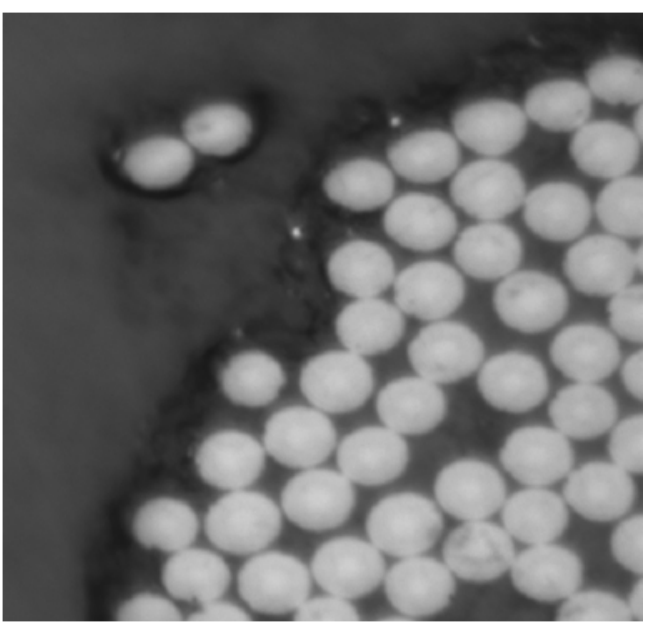

A)

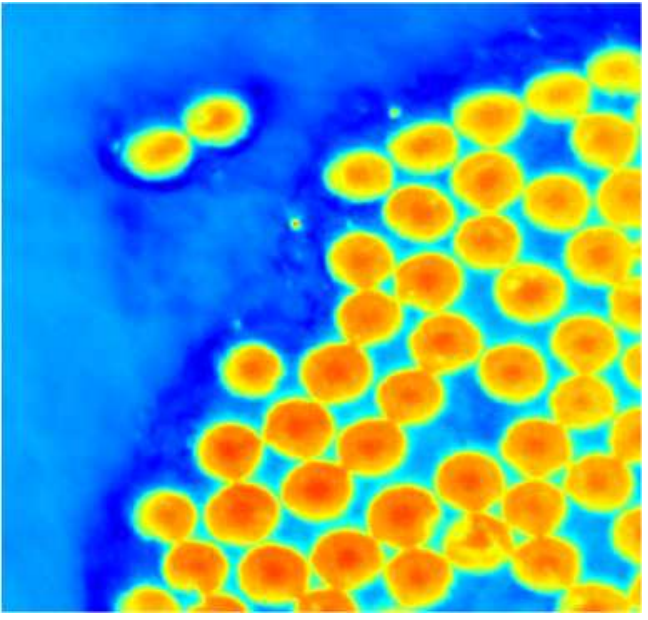

B)

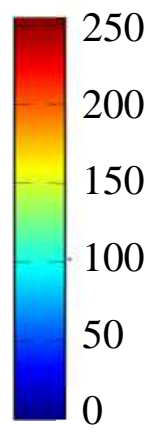

200

150

100

50

Fig. 2. A) Part of a 8 bit micrograph of a carbon fibre reinforced composite cross-section and B) the same image re-coloured to emphasise differences in local colour values. 


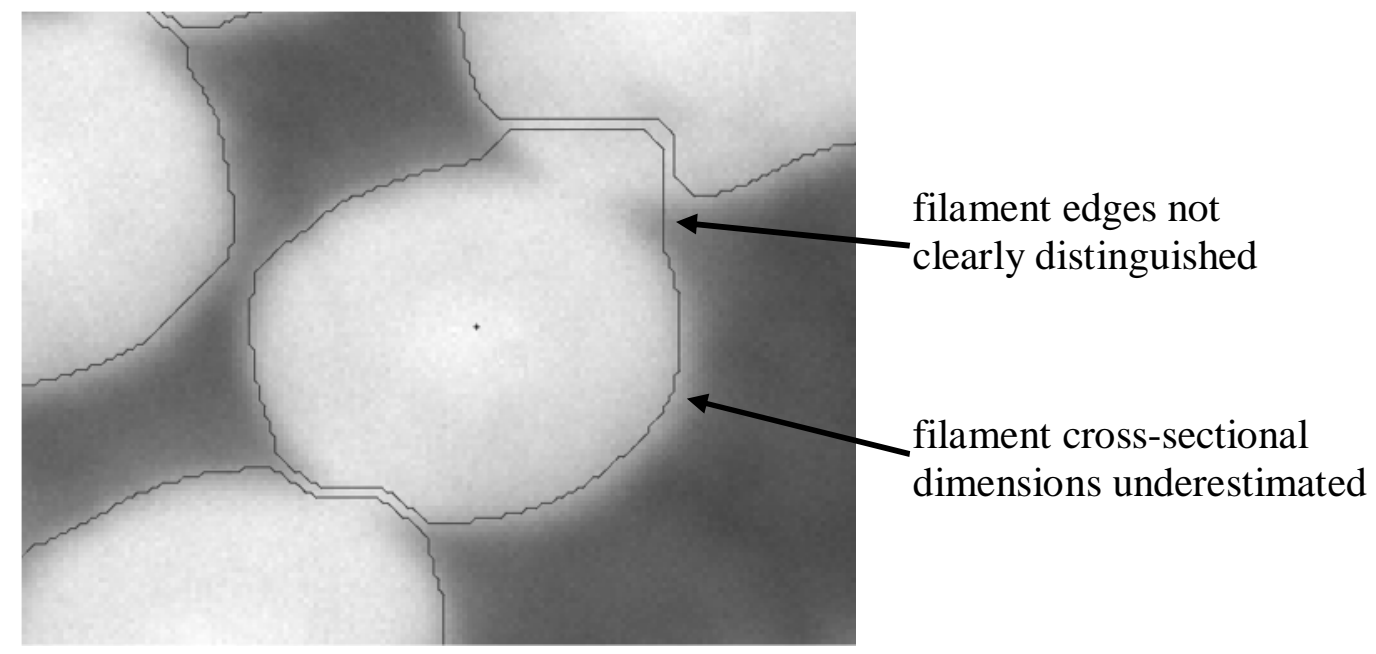

Fig. 3. Magnification of a micrograph of filament cross-sections. Filament boundaries after greyscale thresholding and watershed segmentation are indicated.
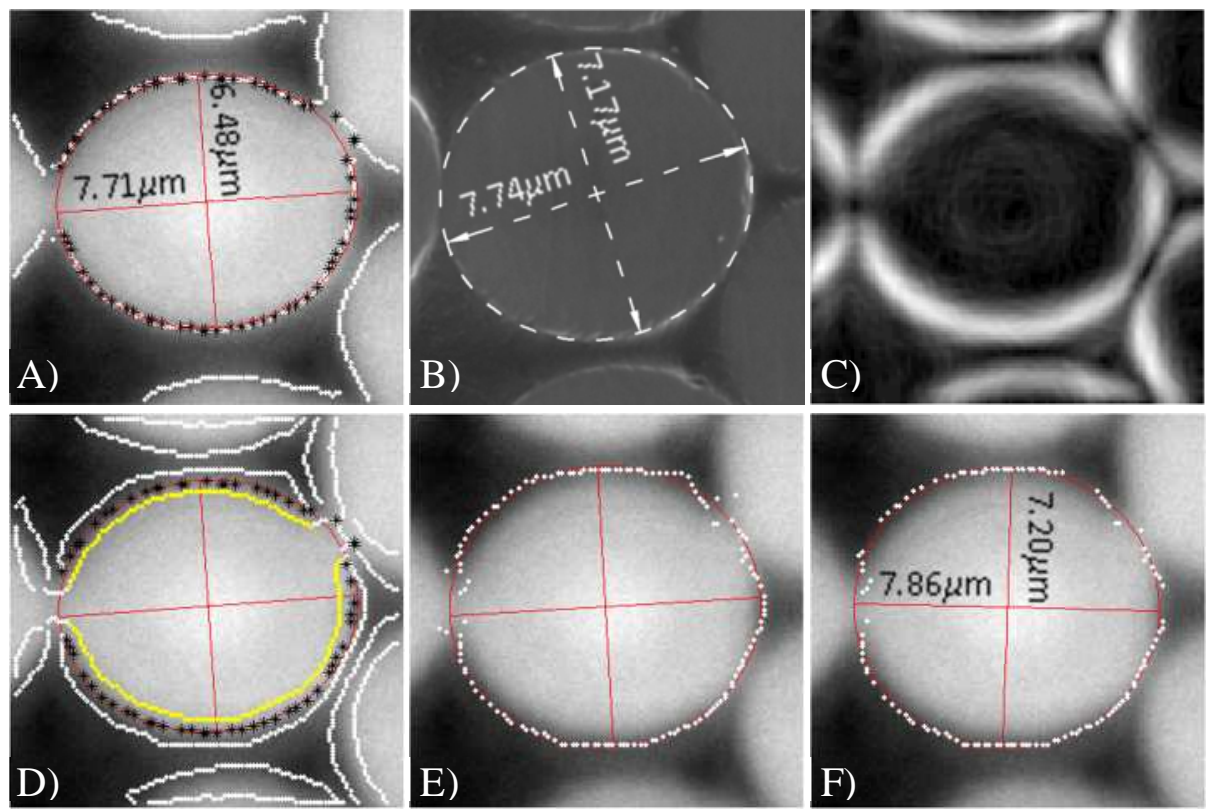

Fig. 4. Single filament cross-section A) Edges detected with the Canny algorithm, B) manually fitted ellipse on SEM image. C) Representation of colour gradients. D) Detected Canny edges (black*) and Canny edges of the gradients (white and yellow dots). E) Initial ellipse fit of fibre boundary with points belonging to edges of touching filaments present. F) Final result of the fitted ellipse after iteratively removing points outside the first ellipse fit. 


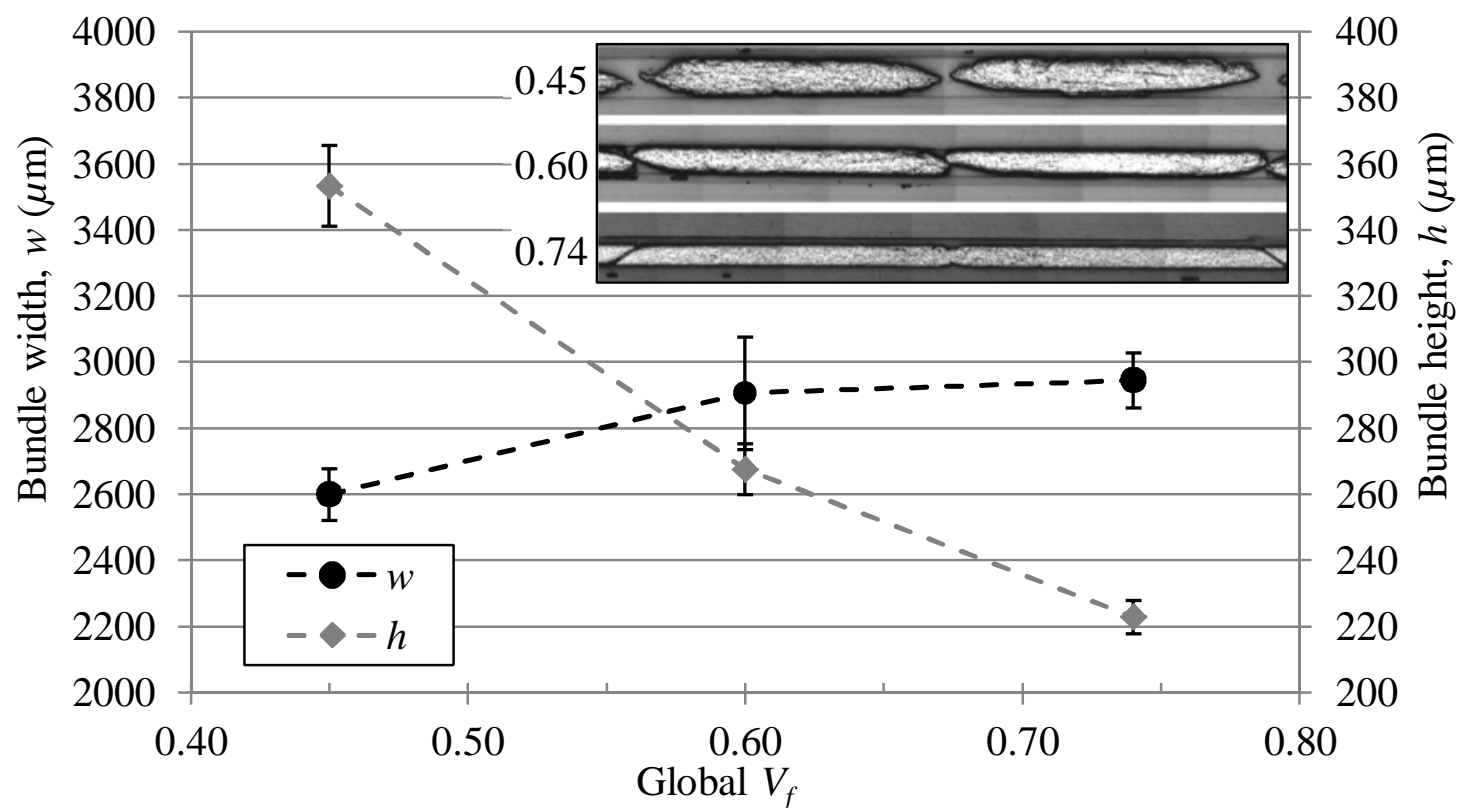

Fig. 5. Fibre bundle height, $h$, and width, $w$, as a function of the global fibre volume fraction, $V_{f}$ of carbon fibre bundles with $12 \mathrm{~K}$ filament count. The error bars show the standard deviation of the measurements.

The inserted image shows corresponding cross-sections of composite specimens produced by RTM.
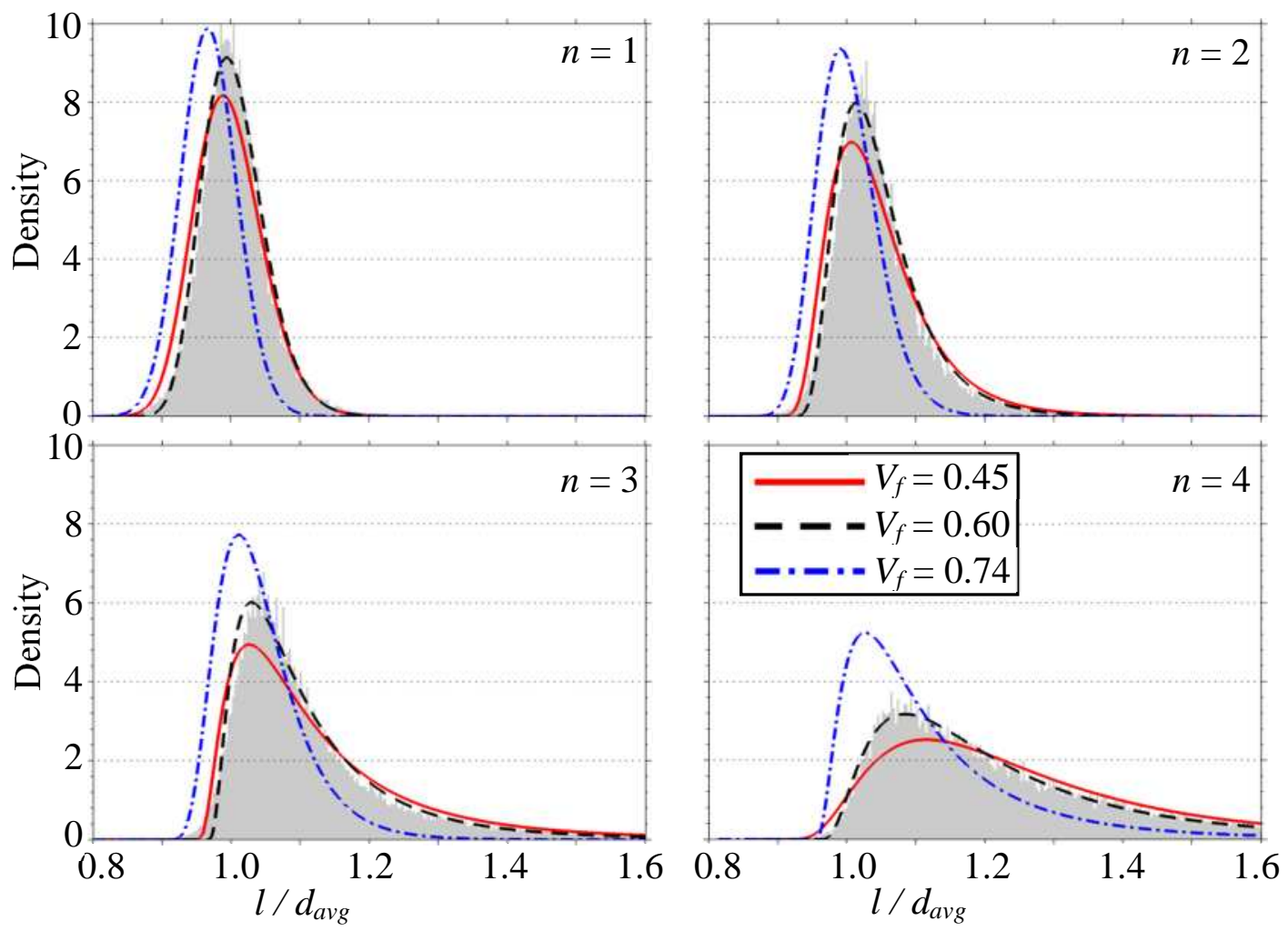

Fig. 6. Distance distribution of a filament to its $n$-th nearest neighbour at different values of $V_{f}$. Distances, $l$, are normalised by the measured average filament diameter, $d_{a v g}$. The actual histograms of the measured distributions for $V_{f}=0.60$ are shown in grey. 


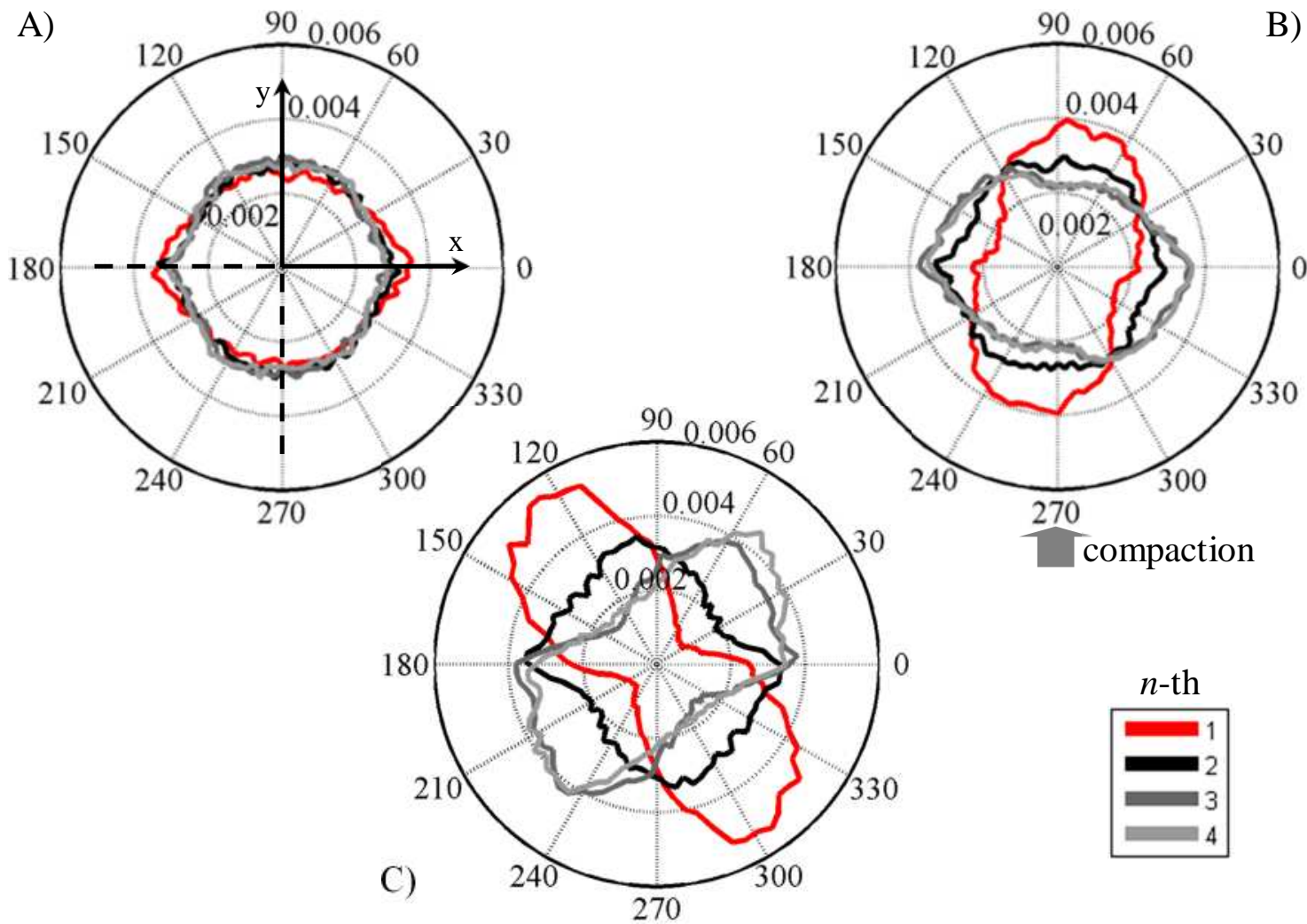

Fig. 7. Angle distributions for the location of the first (red), second to fourth (shades of grey) nearest neighbours. A) global $V_{f}=0.45$; B) global $V_{f}=0.60$; C) global $V_{f}=0.74$. The frequencies plotted on the polar axis are normalised so that the area under the curve is equal to 1.

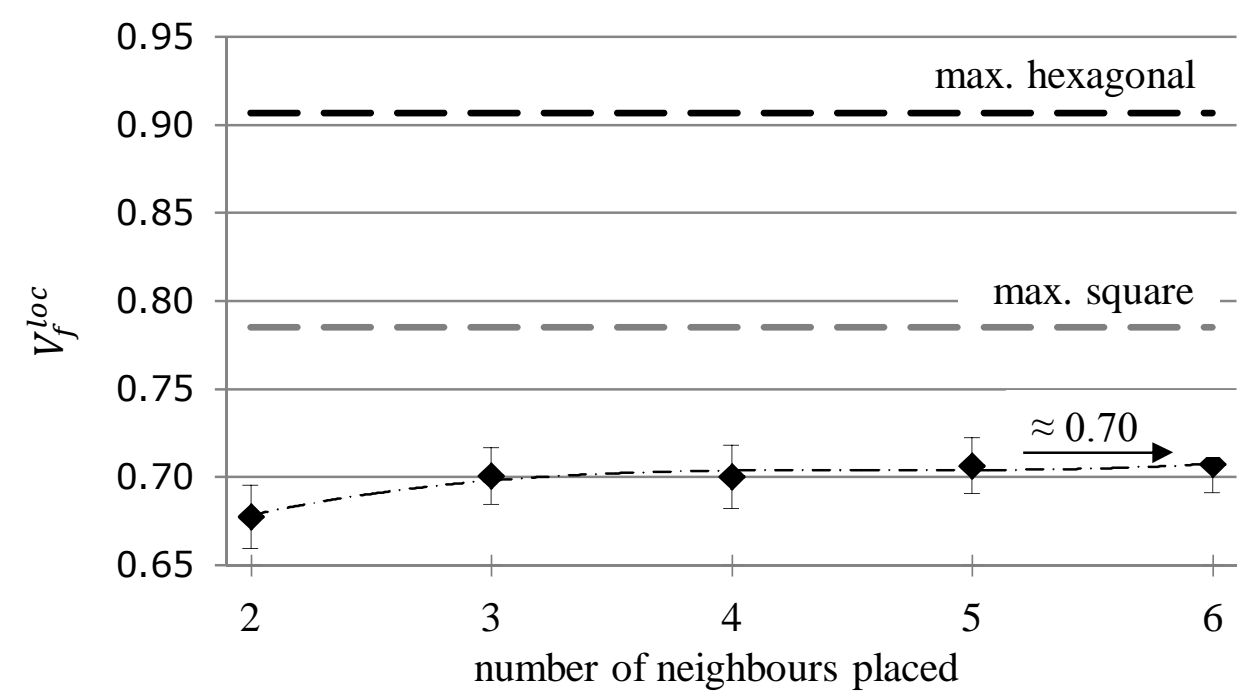

Fig. 8. Jamming limit of the local $V_{f}$ using the fibre placement algorithm by Vaughan and McCarty with varying numbers of neighbour filaments placed. As input, a constant filament radius and constant distance equal to the filament diameter was used such as present in hexagonal and square fibre arrangements at maximum $V_{f}$. 

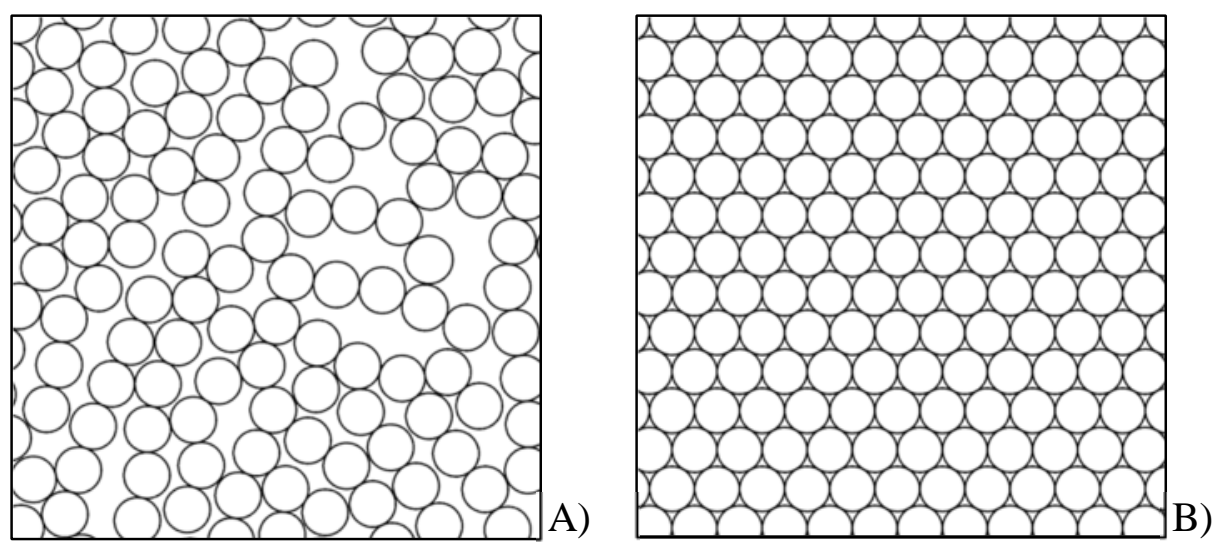

Fig. 9. A) Reconstructed random filament arrangement $\left(V_{f} \approx 0.70\right)$ following the procedure described by

Vaughan and McCarthy [5] with neighbour distance distribution employed equal to the filament diameter.

B) Filament arrangement $\left(V_{f} \approx 0.91\right)$ achieved after additionally incorporating the angle distribution of a hexagonal configuration in the adapted model generator.

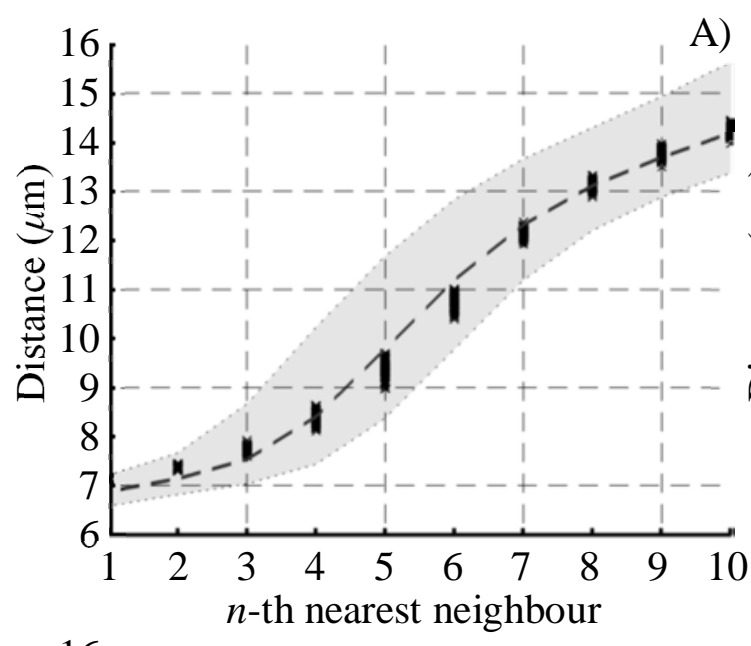

B)
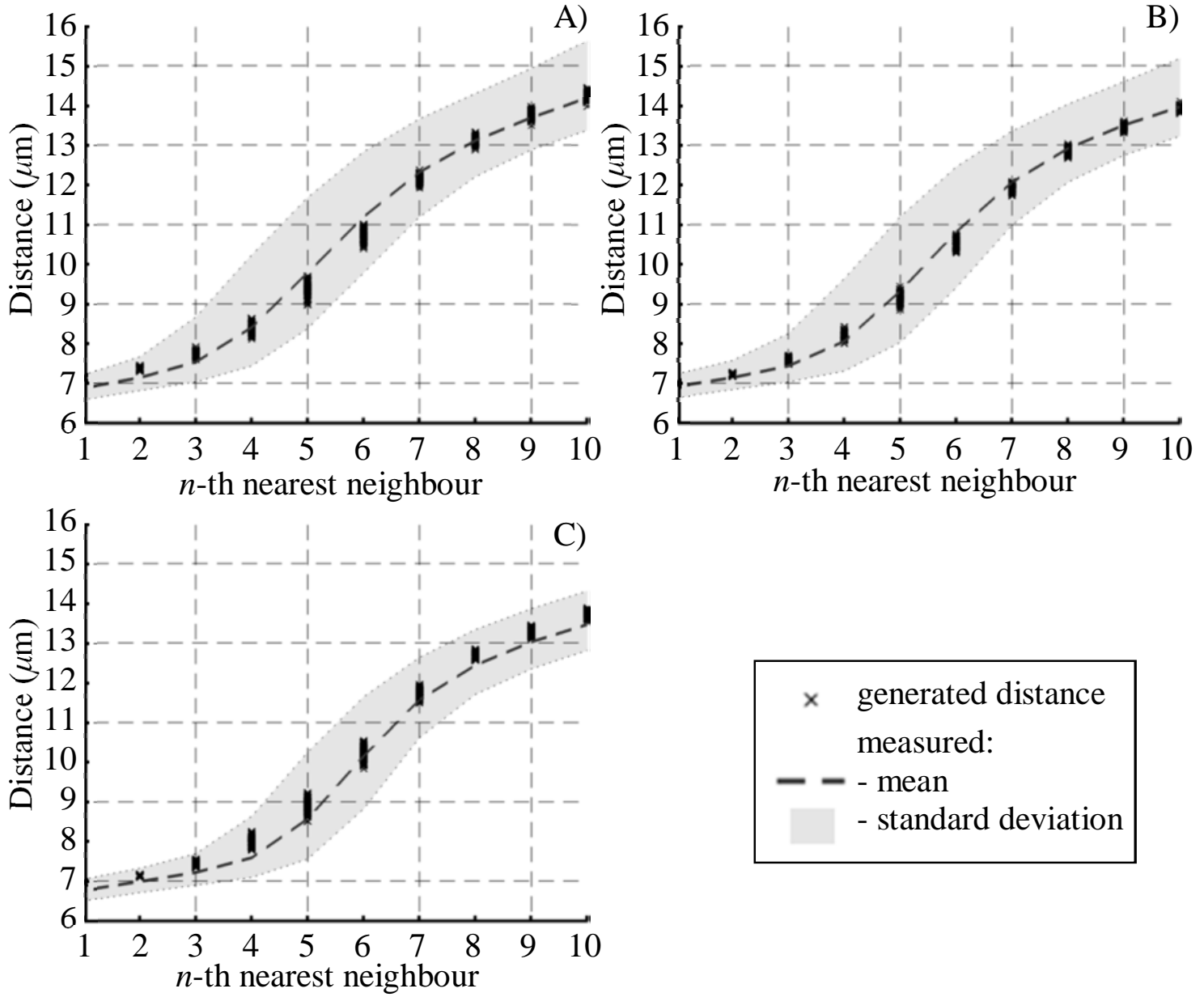

C)

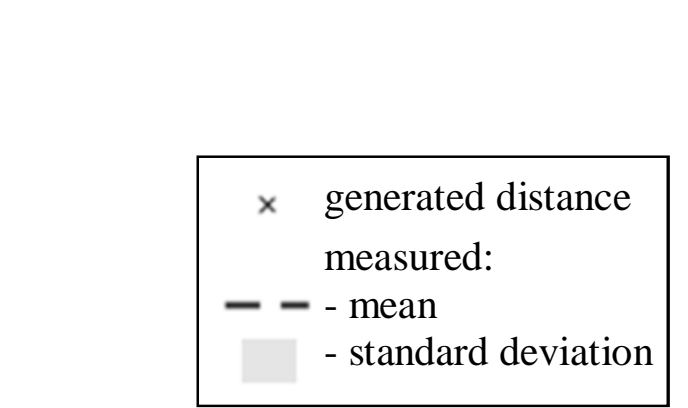

Fig. 10. Generated $n$-th nearest neighbour distances for A) global $V_{f}=0.45$, B) global $V_{f}=0.60$ and C) global $V_{f}=0.74$ compared to the measured lognormal-mean and log-normal standard deviations. 